\title{
Face Recognition Using Principal Component Analysis and Linear Discriminant Analysis on Holistic Approach in Facial Images Database
}

\author{
Satonkar Suhas S. ${ }^{1}$, Kurhe Ajay B. ${ }^{2}$, Dr.Prakash Khanale B. ${ }^{3}$ \\ ${ }^{1}$ Arts, Commerce and Science College, Gangakhed, (M.S.), India, \\ ${ }^{2}$ Shri Guru Buddhiswami College, Purna, (M.S), \\ ${ }^{3}$ Dnyanopasak College, Parbhani, (M.S.),
}

\begin{abstract}
Face recognition system should be able to automatically detect a face in images. This involves extraction of its features and then recognizes it, regardless of lighting, ageing, occlusion, expression, illumination and pose. Principal component analysis and linear discriminant analysis are tested and compared for the face recognition of facial images database. In present paper we have attempted a comparative study of principal component analysis and linear discriminant analysis. The first experiment is used standard face 95 database, local database and pose variation database. The performance of PCA is $100 \%$.The second experiment uses standard Grimance database. The success rate of classification of images is $100 \%$. The value of projection vectors is $0.0076,0.0056,0.0008,0.0036$ and 0.0028 . The graph shows the LDA projection vector, eigenvalues and eigenvectors of these images.
\end{abstract}

Keywords: - Face Recognition, Principal Component Analysis, Linear Discriminant Analysis, Image Processing, Pattern Recognition, Eigenfaces, Face Classification.

\section{INTRODUCTION}

Face recognition have been conducted now for 50 years. Face recognition is widely used in biometric systems. Face recognition is also useful in human computer interaction, virtual reality, database retrieval, multimedia, computer entertainment, information security e.g. operating system, medical records, online banking., biometric e.g. personal identification - passports, driver licenses , automated identity verification border controls, law enforcement e.g. video surveillances, investigation, personal security - driver monitoring system, home video surveillance system. The first paper talking about face recognition can be traced back to the 1950's in psychology [1].The first work concerning automatic face recognition was done in 1970 by Kelly [2], during the 1980's work on face recognition had no progress, but the interest grew rapidly again from the beginning of the 1990's [3] gives some reasons why the research interest increased: real-time hardware became more available, and the importance of surveillance-related applications increased.

There are different types of approaches-holistic approach i.e. appearance-based method, feature-based approach and hybrid approach. In holistic approach the whole face region is taken into account as input data into face detection system. One of the best example of holistic methods are eigenfaces [4] most widely used method for face recognition, In this methods local features such as eyes, nose and mouth are first extracted and their locations and local statistics (geometric and/or appearance) are fed into a structural classifier. A big challenge for feature extraction methods is feature "restoration", this is when the system tries to recover features that are invisible due to large variations, e.g. head pose when we are comparing a frontal image with a profile image[3]. There are different extraction methods, the first is generic methods based on edges, lines, and curves, the second is feature-template-based methods [5] and the third is structural matching methods that take into consideration geometrical constraints on the features. Hybrid approach uses a combination of both holistic and feature-based approaches. Hybrid approach is used in a system developed by Huang [6].

Principal component analysis (PCA) is one of the most popular holistic approach i.e. appearance-based methods used for dimensionality reduction for compression and face recognition problems. Linear discriminant analysis (LDA) is another powerful dimensionality reduction technique which is also known as fisher's discriminant analysis. It has been used widely in many applications such as face recognition [9], image retrieval [10] etc. 


\section{PRINCIPAL COMPONENT ANALYSIS}

Principal component analysis (PCA) is a dimensionality reduction technique which is used for compression and face recognition problems. It is also known as eigenspace projection or karhunen-loeve transformation [11].PCA calculates the eigen vectors of the covariance matrix, and projects the original data onto a lower dimensional feature space, which is defined by eigen vectors with large eigen values. PCA has been used in face representation and recognition where the eigen vectors calculated are referred to as eigen faces.PCA is a useful statistical technique that has found application in fields such as face recognition and image compression, and is a common technique for finding patterns in data of high dimension. It is one of the more successful techniques of face recognition [11].

The benefit of PCA is to reduce the dimension of the data. No data redundancy is found as components are orthogonal. With help of PCA, complexity of grouping the images can be reduced. The application of PCA is made in criminal investigation, access control for computer, online banking, post office, passport verification, medical records etc.

2.1 Methodology find the principal component use the following method [7].

2.1.1 Get the data: Suppose $\mathrm{X} 1, \mathrm{X} 2, \ldots . . \mathrm{X}_{\mathrm{M}}$ is $\mathrm{N} x 1$ Vectors

$$
\mathrm{X}=\underset{\mathrm{i}=1}{\mathrm{M}} \mathrm{M} \sum \mathrm{Xi}
$$

\subsubsection{Subtract the Mean:}

$$
\Phi \mathrm{i}=\overline{\mathrm{X}} \mathrm{i}-\mathrm{X}
$$

2.1.3 Calculating the covariance matrix: form of matrix $\mathrm{A}=\left[\Phi 1, \Phi 2 \ldots \Phi_{\mathrm{M}}\right](\mathrm{N}$ x M matrix $)$ then compute

$$
\mathrm{C}=1 / \mathrm{M} \sum \underset{\mathrm{n}=1}{\Phi_{\mathrm{n}} \Phi_{\mathrm{n}}}={ }^{\mathrm{T}} \mathrm{AA}
$$

2.1.4 Calculating the eigenvector and eigenvalue of the covariance matrix

2.1.5 Choosing components and forming a feature vector: Once eigenvectors are found from the covariance matrix, the next step is to order them by eigenvalue, highest to lowest. This gives the vector. components in order of significance. The eigenvector with the highest eigenvalue is the principle component of the data set. Choose the highest eigenvalue and forming a feature

2.1.6 Deriving the new datasets: Once chosen the components (eigenvectors) that wish to keep in the data and formed a feature vector, imply take the transpose of the vector and multiply it on the left of the original data set, transposed.

Final data $=$ row feature vector $*$ row data adjust

The above formula getting the features of images, the Euclidean distance is calculated between the mean adjusted input image and the projection onto face space. The low values indicate that there is a face and display the face

\section{LINEAR DISCRIMINANT ANALYSIS}

The purpose of discriminant analysis is to classify objects i.e. people, customers, things, etc. into one of two or more groups based on a set of features that describe the objects e.g. gender, age, income, weight, preference score, etc. If one can assume that the groups are linearly separable, one can use linear discriminant model (LDA). Linearly separable suggests that the groups can be separated by a linear combination of features that describe the objects. If only two features, the separators between objects group will become lines. If the number of features is three, the separator is a plane and if the number of features i.e. independent variables is greater than three, the separators become a hyper-plane. linear discriminant analysis (LDA) is commonly used technique for data classification and dimensionality reduction. Linear discriminant analysis is also known as fisher's discriminant analysis and it searches for those vectors in the underlying space that best discriminate among classes. The objective of LDA is to perform dimensionality reduction while preserving as much of the 
class discriminatory information as possible. The goal of LDA is to maximize the between-class scatter matrix measure while minimizing the within-class scatter matrix measure [13].

The Daniel L. Swets and Juyang Weng [10] proposed a two-stage PCA+LDA method, where PCA [11] is used to project images from the original image space to the low-dimensional space and make the within-class scatter non-degenerate. However, the first dimensionality reduction using PCA can also remove the discriminant information that is useful for classification. The most efficient method was proposed by L. Chen, H. Liao, M. Ko, J.Lin and G.Yu.[12], which projects the between-class scatter into the null space of the within-class scatter and chooses the eigenvectors corresponding to the largest eigenvalues of the transferred between-class scatter.

3.1 Methodology The steps in LDA are as follows[24]

3.1.1 Samples for class1 and class2

3.1.2 Calculate the mean of class1 and class2 i.e. Mu1 and Mu2

3.1.3 Covariance Matrix of the first class and second class i.e. S1 and S2

3.1.4 Calculate within-class scatter matrix by using given equation $\mathrm{Sw}=\mathrm{S} 1+\mathrm{S} 2$

3.1.5 Calculate between-class scatter matrix by using given equation $\mathrm{SB}=(\mathrm{Mu} 1-\mathrm{Mu} 2) *(\mathrm{Mu} 1-\mathrm{Mu} 2)$

3.1.6 Calculate the mean of all classes

3.1.7 Compute the LDA projection invSw $=\operatorname{inv}(\mathrm{Sw})$ invSw_by_SB $=\operatorname{invSw} * \mathrm{SB}$

3.1.8 The LDA projection is then obtained as the soluation of the generalized eigen value problem

$$
\mathrm{Sw}^{-1} \mathrm{~S}_{\mathrm{B}} \mathrm{W}=\lambda \mathrm{W}
$$

$$
\mathrm{W}=\operatorname{eig}\left(\mathrm{Sw}^{-1} \mathrm{sb}\right) \text { Where } \mathrm{W} \text { is projection vector }
$$

\section{EXPERIMENTS AND RESULTS}

The experiments were done using PCA, LDA for facial images. The images were obtained from Libor Spacek Collection of facial images[18]. This database includes 7900 colored images of faces of 395 individuals. Each individual has 20 image samples in the database. The database consisits of male and female images of various racial origins. The images are mainly of first year undergraduate students, so the majority of individuals are between 18-20 years old but some under individuals are also present. Some of the individuals has glasses and some of the male individuals have beards. The image format is 24-bit color jpeg in other words 200 x 180 array of pixels and each pixel is represented by 24 bits of RGB color values. The image were recorded with an S-VHS camcorder camera and the lighting is artificial, mixture of tungsten and fluorescent overhead.

\subsection{Experiment I}

Experiment were conducted standard face 95 database and local, pose variation database. The face 95 database contains number of individuals 72. The background consists of a red curtain. Background variation is caused by shadows as subject moves forward. Large head scale and some expression variation. The position of face in image is some translation. All images have same size and the extension of these images jpeg. The local images is created by Digital Camera. The pose variation database is created by Sony (5.1 mp) Digital Camera. These database is available on website http://dsmcsresearch.info.The 71, 10 and 5 face images in the database were tested using PCA. The threshold value of all database is change. The first training set1 include 47 face images (figure 4) and is used to compute the face recogniton based on eigenfaces with Euclidean distance measures technique. The figure 1 shows the sample images of face 95 database. The figure 2 shows the mean images of sample images. The figure 3 shows the mean face of all images and the figure 5 gives the tested input and output image. The second training set2 include 6 face images (figure 9) and is used to compute the face recognition based on eigenfaces with Euclidean distance measures technique. The figure 6 shows the sample images of local database. The figure 7 shows the mean images of sample images. The figure 8 shows the mean face of all images and the figure 10 gives the tested input and output image. The third training set 3 include 3 face images (figure 14) and is used to compute the face recognition based on eigenfaces with Euclidean distance measures technique. The figure 11 shows the sample images of pose variation database. The figure 12 shows the mean images of sample images. The figure 13 shows the mean face of all images and the figure 15 gives the tested input and output image. The success rate of recognition using PCA is $100 \%$. The Table 1 shows the PCA performance. 
Table 1 PCA Performance

\begin{tabular}{|c|c|c|c|c|}
\hline Database & Method & Image Tested & Threshold Value & $\begin{array}{c}\text { Success Rate } \\
\text { (Percentage) }\end{array}$ \\
\hline \multirow{2nnny}{*}{ FACE 95 } & \multirow{3}{*}{ PCA } & 71 & $5.4285 \mathrm{e}+006$ & 100 \\
\cline { 1 - 3 } Local Database & 10 & $2.4198 \mathrm{e}+007$ & 100 \\
\cline { 1 - 3 } $\begin{array}{c}\text { Pose Variation } \\
\text { Database }\end{array}$ & & 5 & $3.4307 \mathrm{e}+007$ & 100 \\
\cline { 3 - 5 } & & & & \\
\hline
\end{tabular}

\subsection{Experiment I Result}

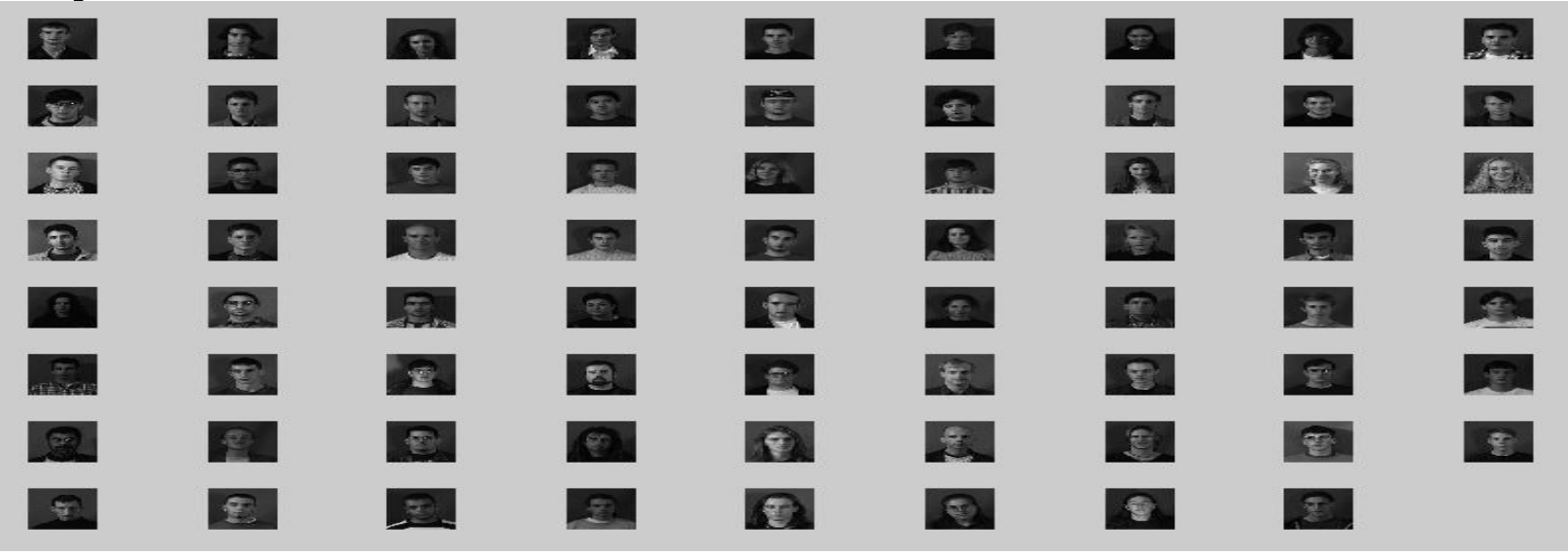

Figure 1 Sample images of face 95 database

\begin{tabular}{|c|c|c|c|c|c|c|c|}
\hline (2) & sis & $a$ & ? & ? & Fi & T & $\mathrm{s}$ \\
\hline Be & s & 舁 & 8 & 줄 & tes & 2 & 贯 \\
\hline 3 & . & Te. & $\sqrt{2}$ & 90 & Px & A & 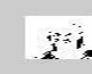 \\
\hline 3 & 5 & 国 & TP & $\vec{x}$ & 5 & 8 & 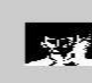 \\
\hline 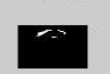 & 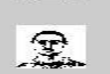 & $\sqrt{1}$ & E? & $P$ & 宗 & 0 & P \\
\hline 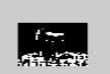 & $\mathcal{E}$ & 5 & 8 & Pु & a & 8 & $\sqrt{35}$ \\
\hline 2 & $F$ & {$[x$} & 7 & 12 & 氺 & 窟 & $\mathbb{B}$ \\
\hline fi & I & ㅊ: & $=$ & 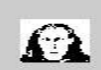 & SO & 8 & 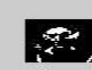 \\
\hline
\end{tabular}

Figure 2 Mean images of face 95 database

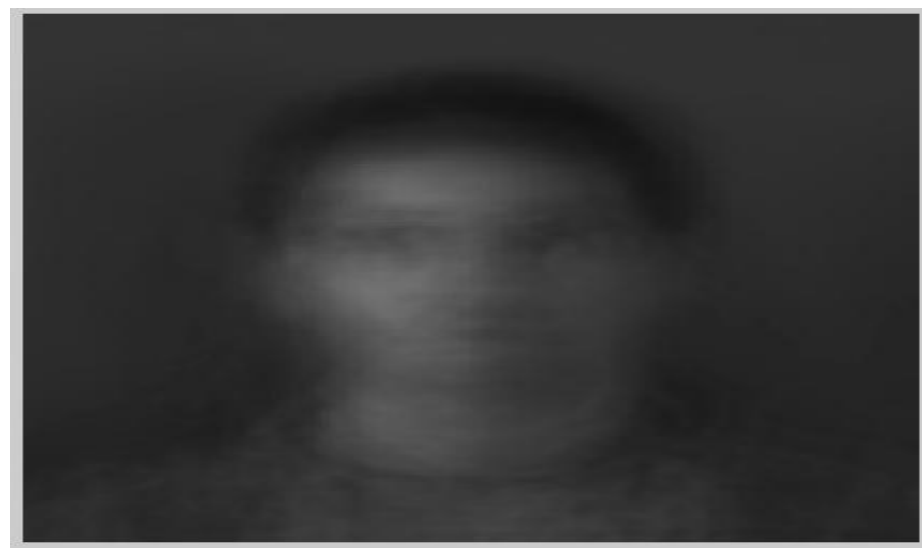

Figure 3 Mean face of sample images 


\begin{tabular}{|c|c|c|c|c|c|c|}
\hline 灌: & 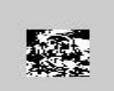 & 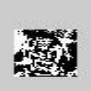 & 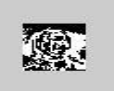 & 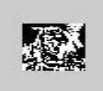 & 㫌 & 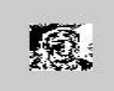 \\
\hline 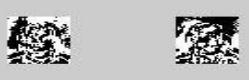 & 窖窑 & 䇾 & 留 & 烮 & 漶 & m \\
\hline 裂 & sis & $\sqrt{2}$ & 6 & 要: & 露 & $\overline{d i d}$ \\
\hline Sis & in & 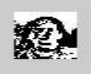 & 可 & T & se & s: \\
\hline 要 & 1) & 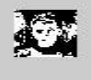 & 2 & ? & $\Omega$ & a \\
\hline
\end{tabular}

Figure 4 Training set 1

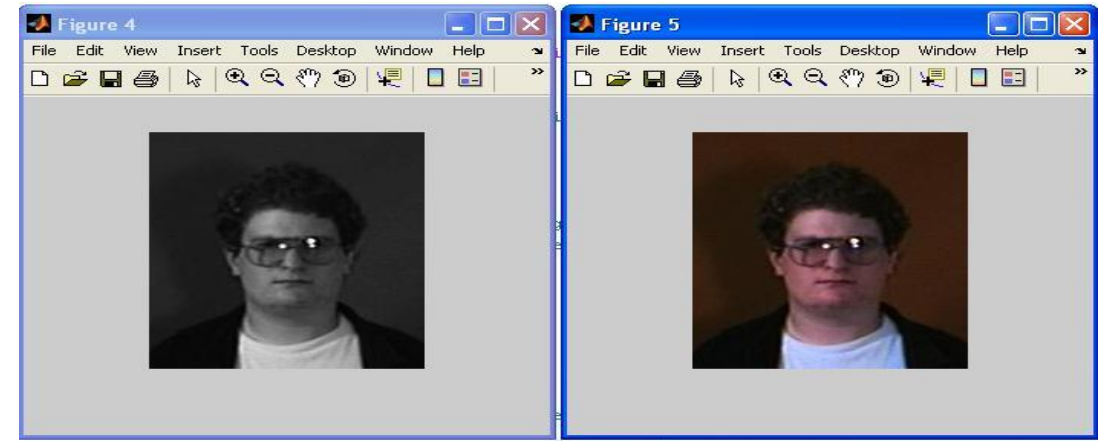

Figure 5 Input Image and Output Image

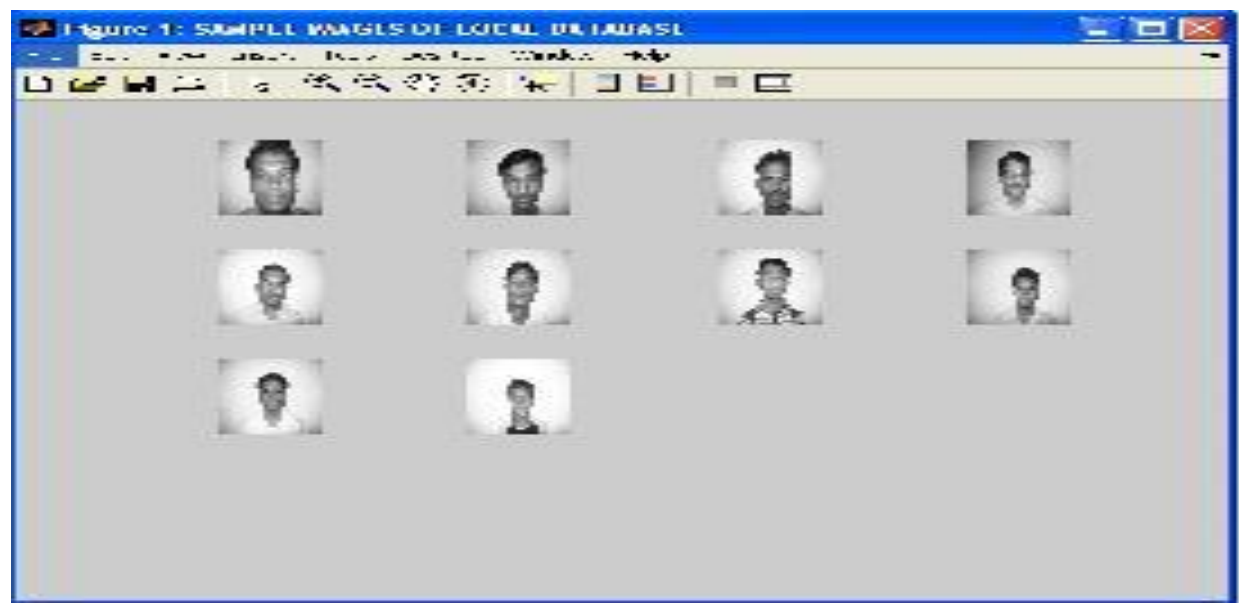

Figure 6 Sample images of Local Database

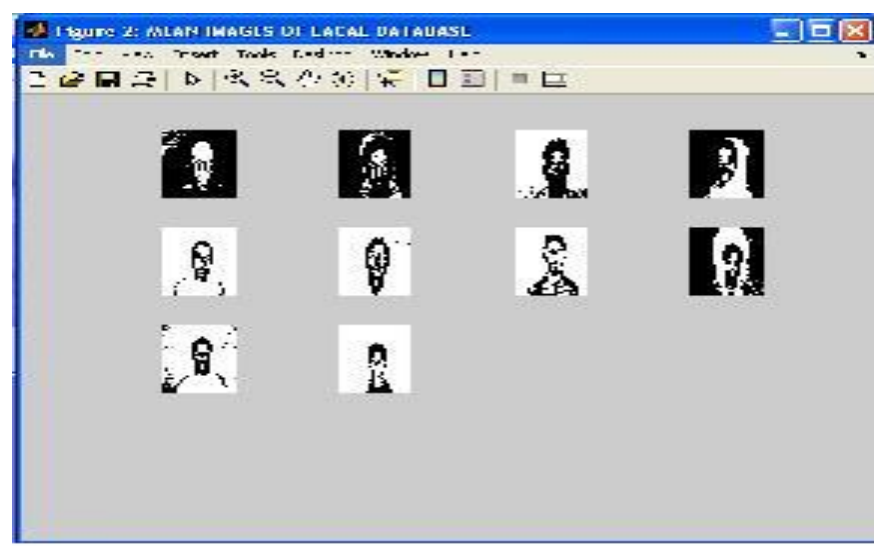

Figure 7 Mean images of Local Database

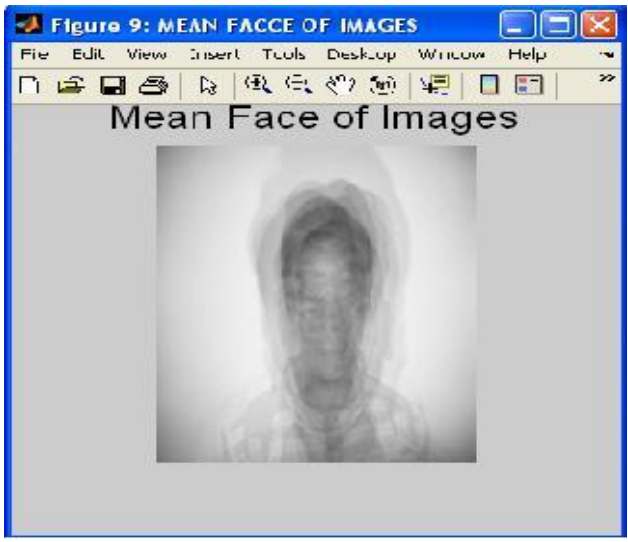

Figure 8 Mean face of sample images 


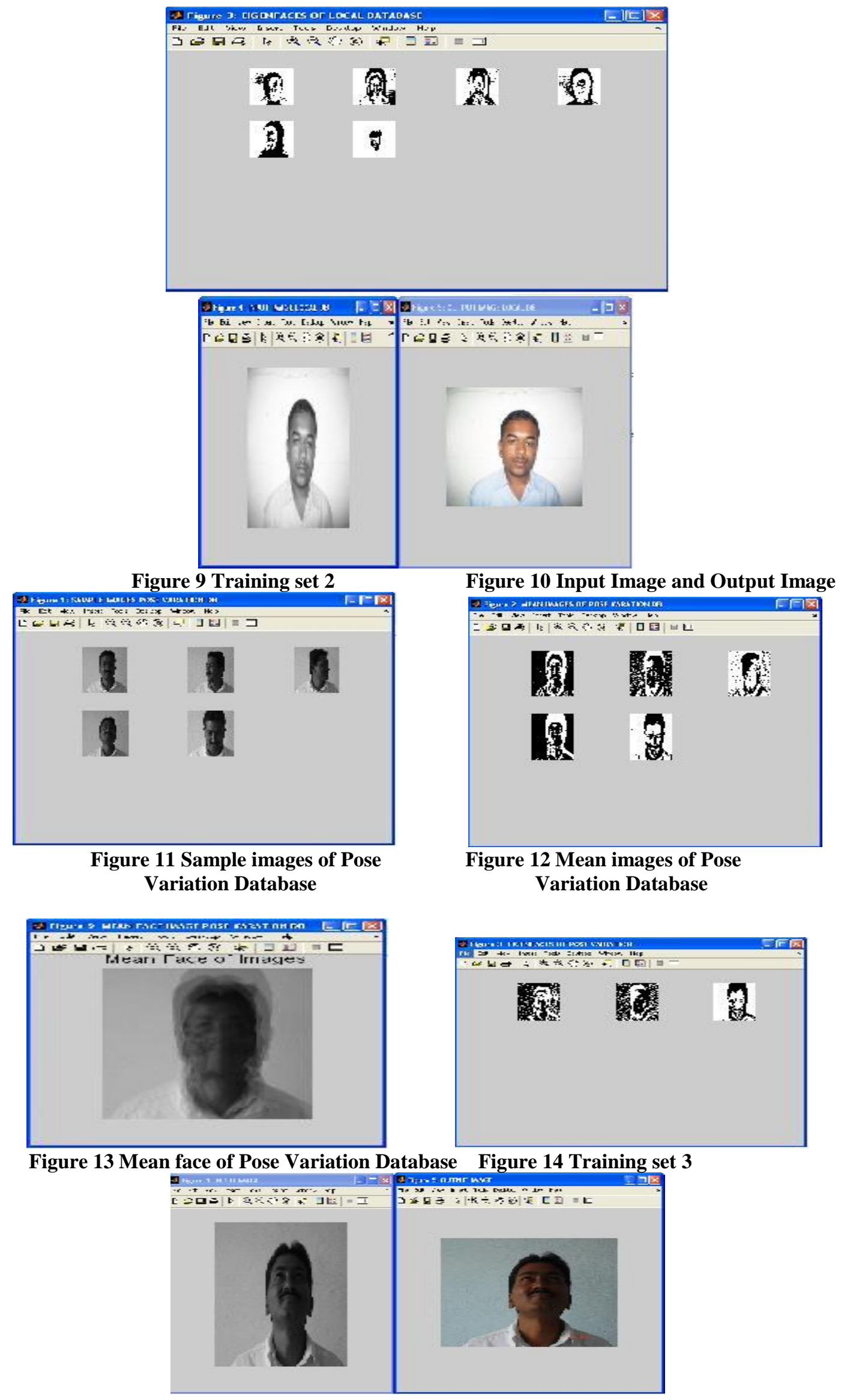

Figure 15 Input Image and Output Image 


\subsection{Experiment II}

Experiment were conducted on Grimance database. The Grimance database contains 18 individuals. The image resolution is $180 \times 200$ pixels. The contains of images of male and female. The background of images is plain in which there are small head scale variation. The lighting variation is very little. The major expression variation. The experiments were used ten images. The class 1 have five images and there is no expression of these images and class 2 used same five images of some expression variation. The sample images of two classes are shown in figure 16. The success rate of classification using LDA is $100 \%$. The graph shows the LDA projection vector, Eigen values and Eigen vectors of these images.

\subsection{Experiment II Result}

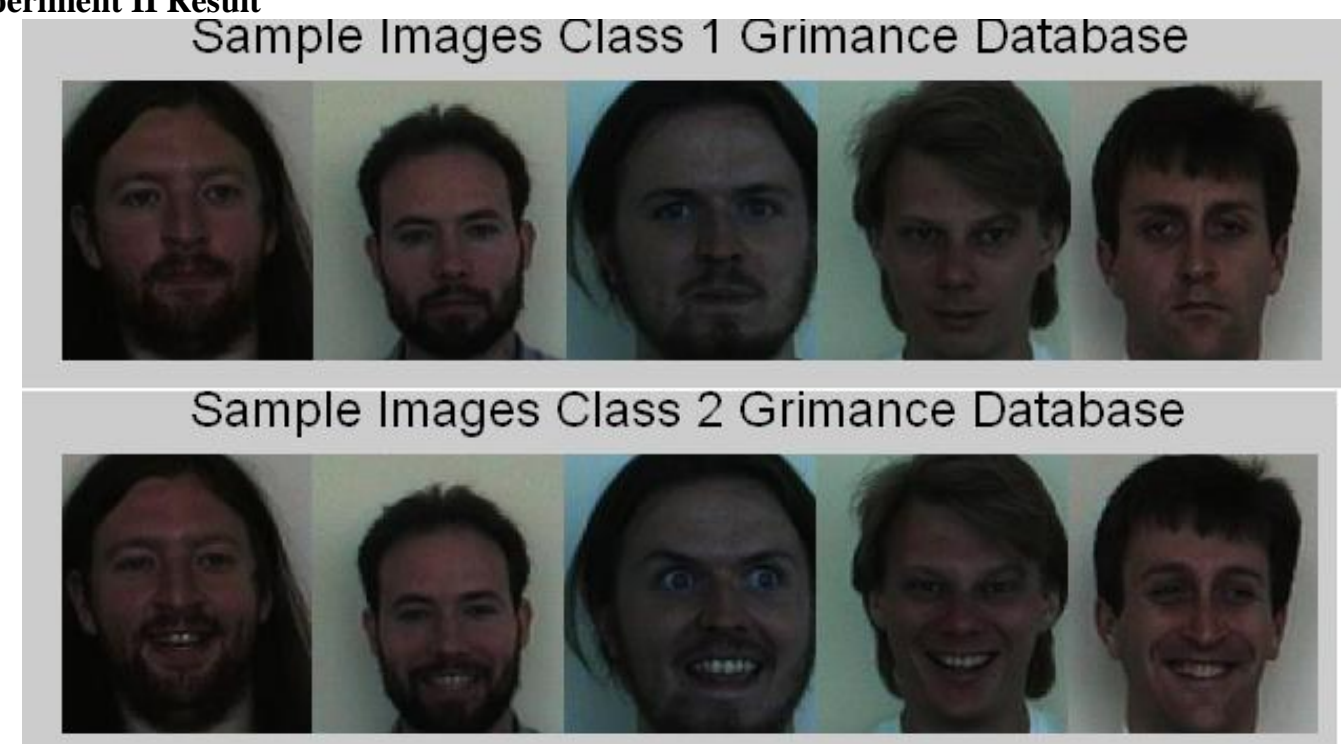

Figure 16 Sample images of two classes

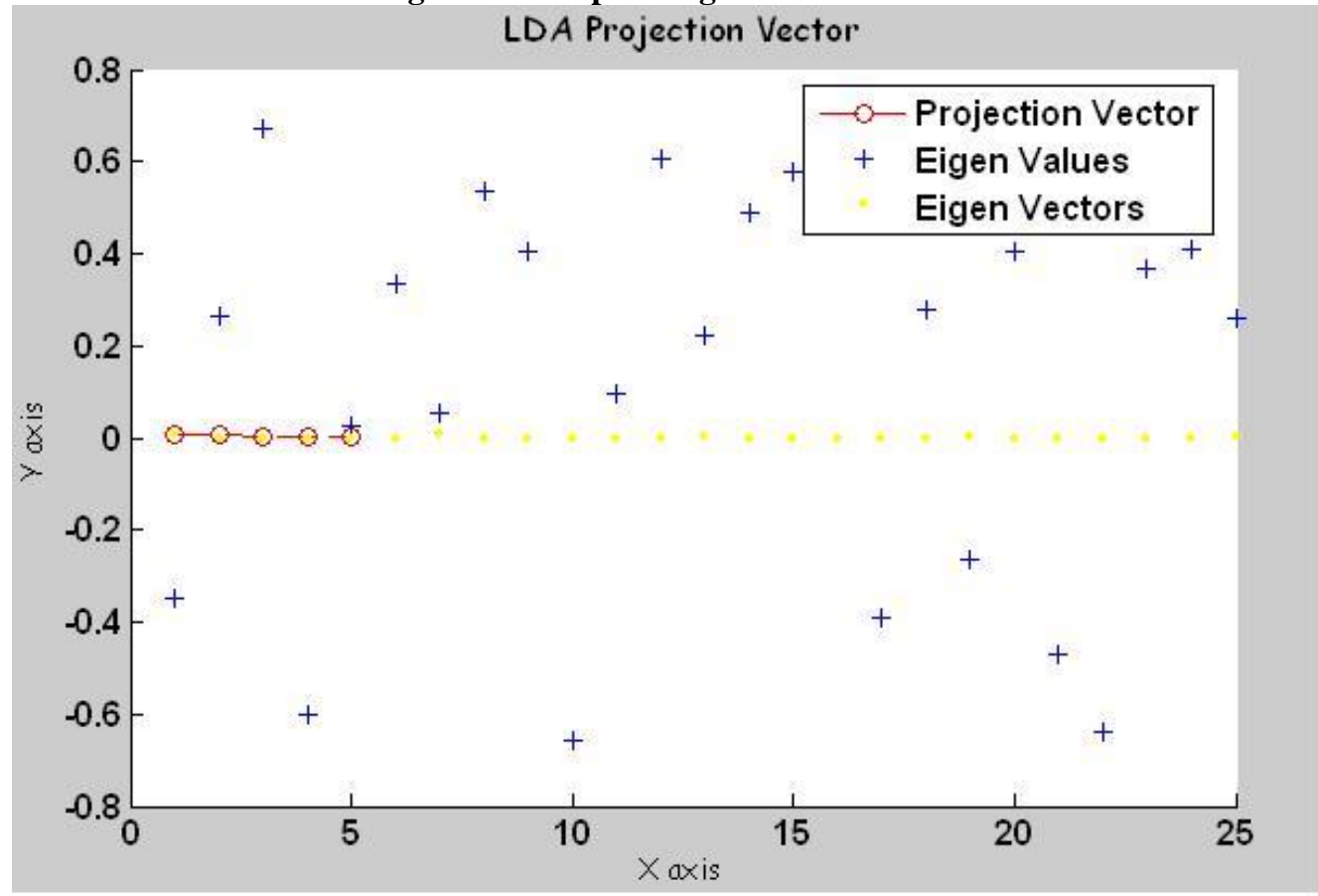

\section{CONCLUSION}

It is observed that a lot of researchers give up working in the face recognition problem due to the inefficiencies of the method used to represent faces. Two holistic approaches principal component analysis and linear discriminant analysis are used in this paper. 
The first experiment use principal component analysis on standard face 95 database. The PCA performance is $100 \%$. The experiment is also tested for locally created poor image quality database and pose variation database and its PCA performance is $100 \%$.

The second experiment use Grimance database images. The graph shows the LDA projection vector and eigenvalues, eigenvectors of these images. The success rate of classification of these samples images is $100 \%$. The value of projection vectors is $0.0076,0.0056,0.0008,0.0036$ and 0.0028 .

\section{ACKNOWLEDGEMENTS}

We are thankful to University Grants Commission (UGC), New Delhi, India, for providing financial Support.

\section{REFERENCES}

[1] Bruner and R.Tagiuri, The perception of people, pages 634-654.Addison-wesley, Second edition, 1954.

[2] M. D. Kelly. Visual identification of people by computer. Ph.D thesis, Stanford University, Stanford, CA, USA, 1971.

[3] W..Zhao, R.Chellappa, P.J..Phillips and A. Rosennfeld,"Face Reconition: A literature Survey.”, ACM Comput.Surv., 35(4): 399-458, 2003.

[4] Matthew A. Turk and Alex P.Pentland, "Face Recognition Using Eigenfaces", Vision and Modeling Group, The Media Laboratory Massachusetts Institute of Technology, CH2983-5/91/0000/0586/\$01.00 1991 , IEEE.

[5] Roberto Bruunelli and Tomaso Poggio, "Face Recognition: Features versus Templates", IEEE Transactions on Pattern analysis and Machine intelligence Vol.15.No.10, October 1993.

[6] J. Huang, B. Heisele, and V. Blanz. "Component-based face recognition with 3d morphable models". In J. Kittler and M. S. Nixon, editors, International Conference on Audio- and Video-Based Biometric Person Authentication (AVBPA-3), volume 2688 of Lecture Notes in Computer Science, pages 2734,Survey, UK, 2003.Springer

[7] L.I.Smith, "A tutorial on Principal Components Analysis".

[8] S.Balakrishnama, A.Ganapathiraju, "Linear Discriminant Analysis- A Brief Tutorial".

[9] P.N. Belhumeour, J.P. Hespanha, and D.J. Kriegman, "Eigenfaces vs.Fisherfaces: Recognition using class specific linear projection", IEEE Transactions on Pattern Analysis and Machine Intelligence, 19(7):711-720, 1997.

[10] Daniel L. Swets and Juyang Weng. "Using discriminant eigenfeatures for image retrieval", IEEE Transactions on Pattern Analysis and Machine Intelligence, 18(8):831-836, 1996

[11] M.Turk and A.Pentland, "Eigenfaces for Recognition", Journal of Congnitive Neuroscience, 3(1), pp.71-86, 1991.

[12] L. Chen, H. Liao, M. Ko, J.Lin and G.Yu. "A new lda-based face recognition system which can solve the small samples size problem", Journal of Pattern Recognition, 33 (10), pp.1713-1726, Oct 2000.

[13] Martinez A.M. and Kak A.C., "PCA versus LDA", IEEE Transactions on Pattern Analysis and Machine Intelligence, Vol. 23, No.2, pp. 228-233, 2001.

[14] Yang J., Yu Y., Kunz W., “An Efficient LDA Algorithm for Face Recognition”, The Sixth International Conference on Control, Automation, Robotics and Vision (ICARCV2000), 2000

[15] L. Sirovich and M. Kirby, "A Low-Dimensional Procedure for the Characterization of Human Faces", J. Optical Soc. Am. Vol.4, no.3, pp.831-836, Aug.1996.

[16] Onsen Toygar, Adnan Acan, "Face Recognition Using PCA, LDA and ICA Approaches on Colored Images", Journal of Electrical and Electronics Engineering, Vol.3, no. 1, pp.735-743, 2003.

[17] Srinivasulu Asadi, Dr.Ch.D.V.Subba Rao, V.Saikrishna, "A Comparative study of Face Recognition with Principal Component Analysis and Cross-Correlation Technique", International Journal of Computer Applications (0975 - 8887), Vol.10, No.8, November 2010.

[18] Libor Spacek., "Description of Libor Spacek collection of Facial Images", 1996, online http://cswww.essex.ac.uk/mv/allfaces/index.html

[19] P.B.Khanale, "Recognition of Marathi Numerals Using Artificial Neural Network", Journal of Artificial Intelligence 3(3): 135-140, 2010.

[20] P.B.Khanale, "Face Recognition Against Variation in Pose and Background", IEEE INTERNATINAL CONFERENCE ON ELECTRO/INFORMATION TECHNOLOGY (EIT 2011), Minnesota State University Mankato, Min, USA,2011.

[21] Satonkar S.S., Kurhe A.B., Khanale P.B "FaceRecognition Methods \& Its Applications", Journal of Emerging Technology and Applications in Engineering, Technologyand Sciences(IJ-ETA-ETS), ISSN: 0974-3588, July 11 Dec 11, Volume 4: Issue 2, Page294-297. 
[22] Satonkar S.S., Kurhe A.B.,Dr.KhanaleP.B., "Challenges in Face Recognition: A Review”, Journal of Advanced Research in Computer Science(IJARCS),ISSN: 09756-5697, July- August IssueIV, Volume 2 No.4 Page No.82-85.

[23] Satonkar S.S., Kurhe A.B., Dr.Khanale P.B., "Face Recognition Using Different Distance Measures Techniques", in the International Journal of Image Processing and Applications (IJIPA), ISSN: 09758178, January-June 2012, Vol.3 No.1, Page 29-36.

[24] Aly A. Farag, Shireen Y.Elhabian, “A Tutorial on Data Reduction”, Octomber 22008. 